\title{
Photovoltaic efficiency of solution-processable organic molecules reached near $10 \%$
}

In the family of organic solar cells with the donor materials of organic molecules (vacuum-deposited or solutionprocessed) and conjugated polymers, solution-processable organic molecules possess the advantages of solutionprocessibility in comparison with the vacuum-evaporated unsoluble organic molecules and definite molecular weight and high purity in comparison with the conjugated polymers. But in early stage of the studies, the researchers pay too more attention to the solubility of the molecules and focused on the molecules based on non-planar triphenylamine (TPA) unit and the molecules based on hyperbranched thiophene oligomers for their good solubility. The non-planar structure of the molecules results in poorer absorption and lower hole mobility, leading to lower photovoltaic efficiency. While the photovoltaic efficiency of conjugated polymers has gained steady progress by designing new polymers with D-A structure, planar structure and side chain engineering (conjugated side chains and electron-withdrawing side chains) and by device optimization.

In recent 5 years, the photovoltaic efficiency of the solution-processable organic molecules as donor materials is also significantly improved due to the introduction of the concepts of planar and D-A structure with side chain engineering in their molecular design. The most distinguish progresses in this field come from UCSB group (Prof. G. C. Bazan and Prof. A. J. Heeger) in USA and Prof. Yongsheng Chen's group at Nankai University, China. Prof. Chen's group developed a series of solution-processable planar and A-D-A structured molecules with different center donor unit and acceptor end groups. They gradually improve the photovoltaic efficiency of the molecules from ca. $3 \%$ to higher than $8 \%$. Recently, they reported a new organic molecule DRCN7T with a strong electro-withdrawing end group (Figure 1). The power conversion efficiency (PCE) of the OSC with DRCN7T as donor, with device optimization in collaboration with researchers at University of Massachusetts (Amherst), Fudan University and South China University of Technology, reached $9.3 \%$ [1]. The high performance was ascribed to the strong absorption and the formation of an optimized morphology with an interpenetrat- ing network consisting of $\sim 10 \mathrm{~nm}$ diameter highly crystalline fibrils of DRCN7T, in combination with the efficient electron transport layer material PFN. In addition, Prof. Yongsheng Chen's group [2] recently reported another A-D-A structured molecule with alkylthio-substituted benzodithiophene (BDT) as central donor unit, and the PCE of the OSC based on the molecule as donor reached near $10 \%$.

The next step for the researchers on the solution-processable organic molecules should consider their possible application after the PCE reached $10 \%$. The poorer filmforming property of the small molecules and the thin active layer thickness of the OSCs based on the small molecules as donor could be challenges for the fabrication of large area OSC devices in comparison with conjugated polymers. Higher hole mobility, better film-forming property and ideal D/A interpenetrating network morphology should draw more attention in future studies of solution-processable organic molecule photovoltaic materials.

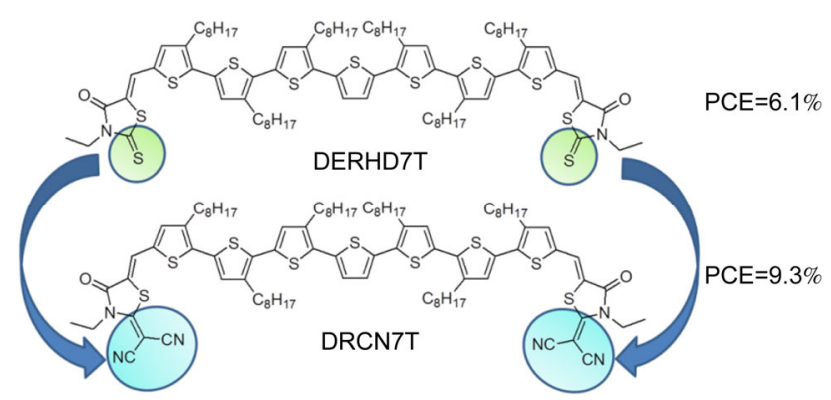

Figure 1 Effect of electron-withdrawing end-group on the photovoltaic performance of the solution-processable organic molecule donor materials.

Yongfang Li

Institute of Chemistry, Chinese Academy of Sciences

1 Zhang Q, Kan B, Liu F, Long G, Wan X, Chen X, Zuo Y, Ni W, Zhang H, Li M, Hu Z, Huang F, Cao Y, Liang Z, Zhang M, Russell TP, Chen Y. Small-molecule-sased solar cells with efficiency over $9 \%$. Nat Photonics, 2015, 9: 35-41

2 Kan B, Zhang Q, Li M, Wan X, Ni W, Long G, Wang Y, Yang X, Feng H, Chen Y. Solution-processed organic solar cells based on dialkylthiol-substituted benzodithiophene unit with efficiency near $10 \%$. J Am Chem Soc, 2014, 136, 15529-15532 